\title{
Responsiveness Among Peers Leads to Productive Disciplinary Engagement
}

\author{
Carolina Alvarado*, Abigail R. Daane ${ }^{\dagger}$, Rachel E. Scherr ${ }^{\dagger}$ and Genaro Zavala* \\ *Physics Education Research and Innovation Group and Department of Physics, Tecnologico de Monterrey, \\ Campus Monterrey, Ave. Eugenio Garza Sada 2501, Monterrey, Nuevo Leon, Mexico 64849 \\ Department of Physics, Seattle Pacific University, Seattle, WA 98119
}

\begin{abstract}
Responsive teaching is the extent to which teachers attempt to understand students' ideas and respond to those ideas in moment-to-moment interactions. We propose that responsiveness does not only apply to teacher-student interactions, but also to interactions among peers. We analyze a small group discussion in a professional development course for K-12 teachers about energy usefulness and identify responsive listening among peers. We observe that learners engage with each other's ideas and further inquire about the reasoning behind those ideas. We claim that responsive listening among peers promotes productive disciplinary engagement which results in the refinement of their understanding about energy.
\end{abstract}

Keywords: Responsiveness, Productive disciplinary engagement, Responsive teaching, Responsive listening. PACS: $01.40 . \mathrm{Fk}, 01.40 . \mathrm{Ha}, 01.40 . \mathrm{J}-$

\section{INTRODUCTION}

Learning to actively listen to students is at the core of a teacher development. [1] Teachers' responsiveness to student's ideas has shown to foster student learning. [2, 3] For this research, we use Pierson's definition of responsiveness: [4] responsiveness reflects the extent to which teachers 'take up' students' thinking and respond to student ideas in their moment-to-moment interactions. Pierson also considers responsiveness as "sensitivity or genuineness in conversation: an attempt to understand what another is thinking displayed in how a conversational partner builds, questions, probes, clarifies, or takes up that which another has said". (p. 25) Responsiveness is an indicator of the depth and quality of teacher-student interaction in a classroom.

We use Pierson code scheme to identify responsiveness since it is well justified, it is methodologically clear and it is frequently cited. Pierson categorized responsiveness into four levels: Low, Medium, High I and High II responsiveness. When teachers do not respond to students' ideas their actions are at a Low responsiveness level (e.g., teachers might repeat their original question without addressing the students' responses). Medium responsiveness uses corrective intervention to redirect students' ideas to fit the canonical content such as vague reformulation. High I responsiveness occurs when a teacher is responsive to the student's idea but the teacher's thinking is on display (e.g., teacher tries to correct a student's misconception by presenting the teacher's reasoning process). High II responsiveness explores student thinking and makes it the focal point of the discussion. In this level teachers are responsive by building on the student's idea and allowing the student thinking to be displayed. For each responsiveness level, Pierson gives detailed descriptions of categories to facilitate the identification of responsiveness in classroom discussion.

Responsiveness is usually attributed to teachers; however, we propose that the idea of responsiveness is not exclusive to teacher-student interactions. In what follows we provide examples that responsive listening occurs during discussions between peers.

A common goal in the learning-teaching process is to foster productive disciplinary engagement (PDE). PDE characterizes the moment-to-moment evolution of disciplinary understanding. Productivity in PDE is defined as the intellectual progress made during group discussions: the extent to which the discussion "gets somewhere." [5] In scientific disciplines, PDE is the refinement of argumentation about learners' claims as well as the generation of new questions in response to the discussion. In our study we seek to show that responsive listening among peers fosters PDE. In the following section we present an analysis of a small group discussion showing that learners productively engage in disciplinary content as a result of being responsive to each other.

For this preliminary study, we observed teachers interacting with each other as peers during the learning process. In future work we will apply this analysis to university student interactions. In this new context we will test for the presence of responsive listening, and whether or not it leads to PDE. 


\section{RESEARCH CONTEXT}

The preliminary study involves K-12 teachers participating in a professional development course at Seattle Pacific University. The course focuses on developing a greater understanding of energy concepts, which include the principle of energy conservation and the concepts of energy usefulness and degradation. [6]In the class session prior to the one we analyze, learners observed and described the energy transfers and transformations in a Stirling engine. The engine was powered by a temperature difference between a cup of hot water (placed below the engine) and the environment. Small groups of learners used Energy Tracking Diagrams [7] to track the transfers and transformations from the hot water to the environment.

In this class session, learners reach a consensus about a diagram in which some thermal energy (TE) in a cup of hot water transforms into kinetic energy that is then transferred to the engine, transformed into TE and finally transferred to the environment. The rest of the TE in the cup transfers directly to the environment. The learners work in small groups to discuss how the $\mathrm{TE}$ in the hot water is different from the TE transferred to the environment at the end of the process. In what follows, we present how responsive listening occurred during this discussion and how learners engaged productively with the physics content during their argumentation.

\section{CASES OF RESPONSIVENESS LEADING TO PRODUCTIVE DISCIPLINARY ENGAGEMENT}

In order to illustrate how responsiveness occurs and show the evolution of the learners' thinking, we analyze three episodes taken from a 30-minute discussion. During these episodes there was no intervention by any of the instructors. Responsiveness among three secondary teachers (Ken, Donna and Larissa) occurs throughout the discussion, not only in the instances shown in these three episodes. The three members of the group show responsiveness to varying degrees during their interaction.

We adapt Pierson's coding scheme for analysis of levels of peer responsiveness. We refer to the characterizations provided by Pierson to justify the responsive listening found. To show PDE we discuss the evolution of the learner's arguments.

\section{Episode 1}

At the beginning of the discussion, the learners list differences in the properties of the gas and liquid states while trying to discuss the differences in energy. Donna and Larissa do not explicitly disambiguate matter and energy. Ken argues for the separation of the properties of matter and energy since they were asked to list the energy differences.

${ }^{1}$ Ken: But the energy is not a particle in the liquid,

${ }^{2}$ Larissa: No but it's

${ }^{3}$ Donna: No but it is in those particles

${ }^{4}$ Ken: But the energy IN the liquid...

In this discussion Ken's intonation for "in" emphasizes that energy is not matter; they are distinct entities. Ken notices both Larissa and Donna don't disambiguate matter and energy during their discussion and response to this. Ken shows a High I level of responsiveness while intervening in the discussion to correct a misconception: they use matter and energy properties interchangeably. Then he asks the following questions to focus the analysis to the energy properties.

${ }^{5}$ Ken: What is the difference of the energy, what can be- if we could observe it, what would be observing, what observable difference in the thermal energy would we see "in" the liquid phase versus the gas phase? Is it hotter? What is it doing?

${ }^{6}$ Donna: I also think that there is also a difference in, what we can get that energy to do or transform into- get some- something that is useful out of it, does that make sense?

${ }^{7}$ Larissa: Do you feel like this T [TE in the hot water] is more useful than this $\mathrm{T}$ [TE in the environment]?

${ }^{8}$ Donna: Yeah, and the reason that I am thinking about that is that this energy [TE in the hot water] is more organized.

${ }^{9}$ Donna: You can transfer enough energy to get some motion out of that. Whereas, the air that, once you get the thermal energy in the air, that spreads out

${ }^{10}$ Larissa: That's distributed.

${ }^{11}$ Donna: It's distributed! I can't go, it is really hard to go back and capture all that to get to do something. Like I can't, I can't just get all the energy, the thermal energy that went into the air and try to run this backwards.

During this discussion, the group analyzes the distribution of the energy related to energy dispersion and occupied volume. Larissa, in line 7, invites Donna to further explain her thinking about the usefulness at both points in the scenario. The use of a substantive probe, or probing question, is one example of a High II level of responsiveness. Though Larissa's question might be interpreted as a closed question, her intonation suggests an effort to create a space where Donna can further explain her reasoning about the usefulness of thermal energy. 
In line 10, we observe a Medium level of responsiveness when Larissa uses a vague reformulation, or an adaptation of one part of Donna's response to impart desired knowledge. After attending to Donna's statement, Larissa suggests that Donna's description aligns with her own ideas about energy distribution.

At the end of this episode, Donna shows a Low level of responsiveness (line 11). She rebroadcasts Larissa's contribution ("It's distributed!"), using an echo that functions as an implicit evaluation of correctness. Donna further states that while the more organized energy goes from the hot water into the environment, it makes the engine spin, but when it gets distributed (less organized) it cannot be used in a reverse way to run the engine again.

After Ken proposes to focus on energy instead of matter, they decide that the quantity of energy remains the same, but the spatial arrangement and the way they can "use" the energy are different in the cup and the environment. Larissa's invitation to Donna to further explain her reasoning leads them to refine their understanding of the energy involved: in other words, her responsiveness increases the group's productive disciplinary engagement. In this episode the learners form their first claim about the scenario: energy flows from the more to the less organized place.

\section{Episode 2}

As the discussion continues, the group introduces another scenario that was discussed during the previous session when the Stirling engine operated using a bag of ice. In both cases (the hot water in the cup and the bag of ice) the engine moved due to the temperature difference between the reservoir (hot water or ice) and the environment.

${ }^{12}$ Larissa: But you were saying, you can't run it backwards [from the air to the reservoir] and yet, when I came in we said, well if we put it in water that's colder than the air -

${ }^{13}$ Donna: So there has to be a temperature difference, but once they get to the point where there's no temperature difference I can't do it, I can't-

${ }^{14}$ Larissa: Yeah, yeah. But isn't that backwards taking somehow the distributed energy in the air and making it more organized?

${ }^{15}$ Donna: To run it backwards?

${ }^{16}$ Larissa: Well if you have cold water than you are (kind of) running it backwards.

After previously establishing that the energy flows from the more to the less organized place, Larissa responds using those ideas mentioned previously and applies them in a new scenario (line 12). Larissa reintroduces the idea presented by Donna that the energy in the air, due to the dispersion or organization, cannot be used to run the engine. Since during the last session it was shown the engine worked with cold water, Larissa proposes to analyze how the energy flows in that scene. We classify this response as an uptake, which is a High II level of responsiveness.

Donna's response to Larissa's (line 13) comment is considered a low level of responsiveness. She explains how the engine works with ice water but does not incorporate Larissa's previous statement, in which "backwards" refers to changing the direction of the energy flow.

Larissa (line 14) repeats her question, this time a more explicit way. This kind of intervention, as the previous one, is considered a low level of responsiveness; it implies Larissa's determination to discuss her proposed idea.

Finally, Donna shows a High II responsiveness level in line 15. After Larissa clarifies her thinking about the energy flow when using ice water, Donna asks for more information to understand Larissa's idea by using a substantive probe.

In Episode 2 Larissa decides to bring the cold water scenario into consideration. Even though it was not part of the activity, Donna responded to her idea and engaged in this new scenario. The consideration of this new scenario lead them to form a second claim: energy flows from the hotter to the colder place (the energy from the environment flows into the cold water through the engine). They consider that the energy in the cold water is "more organized" than the energy in the environment. Larissa questions how the claim developed in Episode 1 applies to the scenario using cold water to power the engine: She describes the cold water situation as "running it backwards," in the sense that the less organized energy in the environment becomes more organized to power the engine. Larissa recognizes a conflict between the two claims ("You were saying... and yet when I came in we said..."). Donna's request for further explanation helps the group decide to explore this new idea even though it was not part of the assigned task. In other words, as a result of Larissa's responsiveness, the group makes intellectual progress on comparing the two different thermal energies involved in the scenario (PDE).

\section{Episode 3}

In Episode 2, the learners establish that energy flows from the environment to the cold water due to a difference of temperature, activating the Stirling engine. But in Episode 1, they had decided that the energy in the environment is less organized than the energy in the hot water, and that energy flows from 
more to less organized. Since they consider cold water energy to be more organized than the energy in the environment, they face two contradictory claims.

${ }^{17}$ Larissa: So if that's the case, is somehow the less organized thermal energy in the air going in to what we're calling more organized thermal energy in the cup?

${ }^{18}$ Donna: Hm. Is your point because the energy direction- energy flow would be going from the air to the cup?

${ }^{19}$ Larissa: The thermal energy is going the otherlike this all [hot water scenario] is- I'm okay with this, but then suddenly... there is this case where energy transfers in the other direction.

Larissa, in line 17, shows a High II level of responsive listening by expressing a contradiction or counterclaim to the group' previous claims. She reveals the contradiction between the energy flow mechanisms. She asks her group to rethink how the TE transfers from the less to the more organized place since they had established that the flow goes the other direction.

Donna responds by inviting Larissa to further explain her concern. Donna, in line 18, shows a High II level of responsiveness corresponding to ask for substantive probe. In other words, Donna tries to understand why Larissa is disturbed about the TE in the air flowing or being transferred into the cold-water filled cup.

Larissa, in line 19, explains how the claims about energy flow are acceptable in the hot water scenario, but are not consistent in the cold water scenario. She attempts to engage the group in the analysis of the contradiction. At this point of the conversation the group decides to refine their claims by analyzing this inconsistency.

In summary, these learners develop two claims about how energy flows in a Stirling engine during their discussion: (1) energy flows from a more organized place to a less organized place because of spatial energy organization and (2) energy flows from hot to cold because of a temperature difference. They progress in their thinking from an initial acceptance of two contradictory mechanisms for energy flow towards an explicit awareness of the contradiction in their claims. This leads them to refine their argumentations of the claim. This productive disciplinary engagement is fostered by the acceptance of and inquiry into each other's ideas.

\section{CONCLUSIONS}

Responsiveness research has primarily explored the activity of teachers' responsive listening to students' reasoning. [8] However, we also observe responsiveness occurring among peers using an adaptation of Pierson's coding scheme. In the episodes shown the learners are responsive listeners to each other, and responsiveness is not exclusive to one participant only.

During this discussion learners did not limit their listening to accepting or declining the proposals during their collaboration. They show a genuine interest to unfold the reasoning of their statements; they question each other as to how they understand the phenomenon analyzed. For example, when one participant introduces the cold water scenario, the others engage in the analysis even though it was not part of the original activity.

In this case study, we observe responsive listening during peer-to-peer interactions. According to our analysis, learners practice responsive listening in different occasions during the activity and promote each other to give further explanations about their reasoning. The acceptance and inquiry of their ideas fosters a refinement in their claims about energy organization. We observe that in this discussion, responsive listening among peers leads to PDE.

\section{ACKNOWLEDGMENTS}

We are grateful for discussions of this work with the members of Seattle Pacific University's Physics Education Research Group and Ben Geller. This work was supported in part by Tecnologico de Monterrey through grant number CAT140, and by the National Science Foundation under Grant No. 0822342.

\section{REFERENCES}

1. S. Empson and V. Jacobs, in International Handbook of Mathematics Teacher Education, edited by D. Tirosh and T. Wood, (Sense Publishers, Toterdam, The Netherlands, 2008), Vol. II, pp. 257-281.

2. D. Hammer, Cognition and Instruction 15 (4), 485-529 (1997).

3. M. C. O'Connor and S. Michaels, Anthropology \& Education Quarterly 24 (4), 318-335 (1993).

4. J. L. Pierson, The Relationship Between Patterns of Classroom Discourse and Mathematics Learning, University of Texas at Austin, 2008.

5. R. A. Engle and F. R. Conant, Cognition and Instruction, 20 (4), 399-483 (2002).

6. A. R. Daane, V. Stamatis and R. E. Scherr, "Learner understanding of Energy Degradation" (this volume).

7. R. E. Scherr, H. G. Close, E. W. Close and S Vokos, Physical Review Special Topics - Physics Education Research 8 (2), 020115 (2012).

8. A. C. Maskiewicz and V. A. Winters, Journal of Research in Science Teaching 49 (4), 429-469 (2012). 